\title{
EXTENDING THE CONCEPT OF GENUS TO DIMENSION $N$
}

\author{
CARLO GAGLIARDI ${ }^{1}$
}

\begin{abstract}
Some graph-theoretical tools are used to introduce the concept of "regular genus" $\mathcal{G}\left(M_{n}\right)$, for every closed $n$-dimensional PL-manifold $M_{n}$. Then it is proved that the regular genus of every surface equals its genus, and that the regular genus of every 3-manifold $M_{3}$ equals its Heegaard genus, if $M_{3}$ is orientable, and twice its Heegaard genus, if $M_{3}$ is nonorientable. A geometric approach, and some applications in dimension four are exhibited.
\end{abstract}

1. Definitions and notations. Let $\Gamma=(V, E)$ be a regular multigraph of degree $n+1, \gamma: E \rightarrow \Delta_{n}=\{i \in \mathbf{Z} \mid 0 \leqslant i<n\}$ an $(n+1)$-line-colouring of $\Gamma$ [Ha, p. 133]. Such a pair $(\Gamma, \gamma)$ is said to be an $(n+1)$-coloured graph. For every subset $\mathscr{B}$ of $\Delta_{n}, \Gamma_{\mathscr{B}}$ will denote the subgraph $\left(V, \gamma^{-1}(\mathscr{B})\right)$; further, for every colour $c \in \Delta_{n}, \hat{c}$ will denote the set $\Delta_{n}-\{c\}$.

A 2-cell imbedding i: $|\Gamma| \rightarrow F[\mathbf{W}$, p. 40] of an $(n+1)$-coloured graph $(\Gamma, \gamma)$ on a closed surface $F$ is called a regular imbedding if its regions are bounded by 2-coloured cycles. Moreover, $\mathbf{i}$ is called a strongly-regular imbedding if there exists a cyclic permutation $\varepsilon=\left(\varepsilon_{0}, \ldots, \varepsilon_{n}\right)$ of $\Delta_{n}$, so that each region is bounded by a component of one of the subgraphs $\Gamma_{\left\{\varepsilon_{i}, \varepsilon_{i+1}\right\}}, i$ being an integer modulo $n+1$.

It is easy to see that every strongly-regular imbedding is regular. Conversely it is proved in $\left[\mathbf{G}_{\mathbf{4}}\right]$ that every regular imbedding of a 3- or 4-coloured graph is strongly regular. This is not true in general, as it is easy to check.

In the present work, we only consider strongly-regular imbeddings, as they seem to apply better to the geometric situation we wish to represent.

For the sake of conciseness, we shall always omit the word "strongly". So we shall simply call regular the strongly-regular imbeddings of $\left[\mathbf{G}_{\mathbf{4}}\right]$.

A pseudocomplex [HW, p. 49] $K=K(\Gamma)$ of dimension $n$ can be associated to every $(n+1)$-coloured graph, so that $\Gamma$ becomes its dual 1-skeleton; for the construction, compare $\left[\mathbf{G}_{2}\right]$. If, for every colour $c \in \Delta_{n}$, the subgraph $\Gamma_{\hat{c}}$ is connected, then $K(\Gamma)$ has exactly $n+1$ vertices, and both $K$ and $\Gamma$ are said to be contracted.

Now let $M$ be a closed $n$-manifold, $(\Gamma, \gamma)$ a contracted $(n+1)$-coloured graph, such that the space $|K(\Gamma)|$ of its associated complex is homeomorphic with $M$; then

Received by the editors July 23, 1979 and, in revised form, December 19, 1979.

AMS (MOS) subject classifications (1970). Primary 57C15, 57C99; Secondary 05C10, $05 \mathrm{Cl}$.

Key words and phrases. PL-manifold, genus, Heegaard genus, Heegaard diagram, multigraph, 2-cell imbedding, line-colouring, $(n+1)$-coloured graph, regular imbedding, regular genus, crystallisation, pseudocomplex, contracted complex.

${ }^{1}$ This work was performed under the auspices of the G.N.S.A.G.A. of the C.N.R. (National Research Council) of Italy. 
$(\Gamma, \gamma)$ is called a crystallisation and $K$ a contracted triangulation of $M$. A theorem of Pezzana $\left[\mathbf{P}_{\mathbf{1}}\right],\left[\mathbf{P}_{\mathbf{2}}\right]$ assures that every closed $\boldsymbol{n}$-manifold admits a contracted triangulation, and therefore it can be represented by a crystallisation. A faster construction for the case of a 2-fold branched covering of $S^{3}$ is shown in [F].

By a Heegaard splitting of genus $n$ for a closed 3-manifold $M$ is meant a pair $\left(\mathbb{Q}, \mathbb{Q}^{\prime}\right)$ of handlebodies of genus $n$ [He, p. 15], such that $\mathbb{Q} \cap \mathbb{Q}^{\prime}=\partial \mathbb{Q}=\partial \mathbb{Q}^{\prime}$, and $\mathbb{Q} \cup \mathbb{Q}^{\prime}=M$. Their common boundary $F=\partial \mathscr{Q}=\partial \mathbb{Q}^{\prime}$ is called the Heegaard surface of the splitting. Compare also [ST] and [M].

All maps and spaces considered in this work are in the polyhedral category; we refer to [GI] for the main tools about PL-topology and to [Ha] for the used graph-theoretical arguments.

2. The regular genus of a closed $n$-manifold. Let $(\Gamma, \gamma)$ be a connected $(n+1)$ coloured graph. It is shown in $\left[\mathbf{G}_{4}\right]$ that $\Gamma$ admits exactly $n ! / 2$ regular imbeddings in some orientable (resp. nonorientable) surface, iff $\Gamma$ is a bipartite (resp. nonbipartite) graph. Besides, no regular nonorientable (resp. orientable) imbedding may exist for a bipartite (resp. nonbipartite) $(n+1)$-coloured graph. So the following definition is well posed.

Definition 1. By the regular genus $\rho(\Gamma)$ of $(\Gamma, \gamma)$ we mean the smallest integer $n$, such that $\Gamma$ regularly imbeds on the (orientable or nonorientable) closed surface of genus $n$.

As bipartite (resp. nonbipartite) graphs admit only orientable (resp. nonorientable) such imbeddings, then $\rho(\Gamma)$ will be orientable or nonorientable according to $\Gamma$ being a bipartite or a nonbipartite graph.

Now let $M$ be a closed $n$-manifold, and $\Subset=\Subset(M)$ the class of all the crystallisations of $M$. Observe that $\mathbb{E}$ is nonempty by Pezzana's theorem. Further, any two crystallisations of $\mathbb{E}$ are joined by a finite sequence of moves, described in [FG].

Definition 2. With the prior notations, set

$$
\mathcal{G}(M)=\min \{\rho(\Gamma) \mid(\Gamma, \gamma) \in \mathbb{E}(M)\} .
$$

We shall call $\mathcal{G}(M)$ the regular genus of the $n$-manifold $M^{2}{ }^{2}$

3. The main results.

THEOREM. (A) Let $M_{2}$ be a closed surface, and $\mathrm{g}\left(M_{2}\right)$ its genus. Then $\mathcal{G}\left(M_{2}\right)=$ $g\left(M_{2}\right)$.

(B) Let $M_{3}$ be a closed 3-manifold, and $\mathcal{H}\left(M_{3}\right)$ its Heegaard genus. ${ }^{3}$ Then

(i) $\mathcal{G}\left(M_{3}\right)=\mathscr{H}\left(M_{3}\right)$ if $M_{3}$ is orientable;

(ii) $\mathcal{G}\left(M_{3}\right)=2 \mathcal{H}\left(M_{3}\right)$ if $M_{3}$ is nonorientable.

\footnotetext{
${ }^{2}$ A similar concept can be defined for every space representable by $(n+1)$-coloured graphs.

${ }^{3}$ The Heegaard genus of a 3-manifold $M_{3}$ is defined as the smallest integer $h$, such that $M_{3}$ admits a Heegaard splitting of genus $h$. Observe that the Heegaard surface of the splitting has genus $h$, if orientable, or $2 h$, if nonorientable.
} 
Proof of Theorem A. Every 3-coloured graph (in particular every surface-crystallisation) represents a closed surface $M_{2}$ if and only if it regularly imbeds in $M_{2}$ [ $\mathbf{G}_{4}$, Corollary 4]. Moreover, this imbedding is unique, up to equivalences [ $\mathbf{G}_{4}$, Proposition 3]. Thus $\mathcal{G}\left(M_{2}\right)=g\left(M_{2}\right)$, for every closed surface $M_{2}$.

The proof of Theorem (B) needs some notations and two lemmas.

Let $M_{3}$ be a closed 3-manifold and $\left(Q, Q^{\prime}\right)$ a Heegaard splitting of genus $n$ for $M_{3}$. By a Heegaard diagram arising from $\left(\mathscr{Q}, \mathbb{Q}^{\prime}\right)$ is meant a triple $(F, \mathbf{x}=$ $\left.\left(x_{1}, \ldots, x_{n}\right), \mathbf{y}=\left(y_{1}, \ldots, y_{n}\right)\right)$, where $F=\partial \mathscr{Q}=\partial Q^{\prime}$ is the Heegaard surface of the splitting, and $x, y$ are two disjoint systems of curves on $F$, satisfying the following properties.

(a) each curve $x_{i}$ (resp. $y_{i}$ ) bounds a 2-ball $\mathbf{X}_{i}$ of $\mathbb{Q}$ (resp. $\mathbf{Y}_{i}$ of $\mathbb{Q}^{\prime}$ ).

(b) $\mathbb{Q}$ (resp. $\mathbb{Q}^{\prime}$ ), cut open along the 2-balls $\mathbf{X}_{i}\left(\operatorname{resp} . \mathbf{Y}_{i}\right), i=1, \ldots, n$, is a 3-ball.

Now let $(\Gamma, \gamma)$ be a crystallisation of $M_{3}$. As shown in [ $\mathbf{G}_{4}$, Proposition 11], for every pair of colours $\alpha, \beta \in \Delta_{3}, \Gamma$ admits a regular imbedding $i_{\alpha \beta}:|\Gamma| \rightarrow F_{\alpha \beta}$, where $F_{\alpha \beta}$ is a Heegaard surface of $M_{3}$, determining the splitting $\left(Q_{\alpha \beta}, Q_{\alpha \beta}^{\prime}\right)$. Also let $\xi_{0}, \ldots, \xi_{n}$ be the components of $\Gamma_{\{\alpha, \beta\}}, \xi_{0}^{\prime}, \ldots, \xi_{n}^{\prime}$ the ones of $\Gamma_{\left\{\alpha^{\prime}, \beta^{\prime}\right\}}, \alpha^{\prime}, \beta^{\prime}$ being the remaining two colours.

Set $x_{i}=\mathbf{i}_{\alpha \beta}\left(\xi_{i}\right)$ and $y_{i}=\mathbf{i}_{\alpha \beta}\left(\xi_{i}^{\prime}\right)$, for $i \in \Delta_{n}$; finally let

$$
\mathbf{x}(\hat{j})=\left\{x_{0}, \ldots, \hat{x}_{j}, \ldots, x_{n}\right\}, \quad \mathbf{y}(\hat{k})=\left\{y_{0}, \ldots, \hat{y}_{k}, \ldots, y_{n}\right\} .
$$

LEMMA 3. For every $j, k \in \Delta_{n}$, the triple $\left(F_{\alpha \beta}, \mathbf{x}(\hat{j}), \mathbf{y}(\hat{k})\right)$ is a Heegaard diagram of $M_{3}$, arising from the splitting $\left(\mathbb{Q}_{\alpha \beta}, \mathbb{Q}_{\alpha \beta}^{\prime}\right)$.

Proof. It is shown in $\left[P_{2}\right]$, and subsequently in $\left[\mathbf{G}_{4}\right]$ that $\left(\mathbb{Q}_{\alpha \beta}, \mathbb{Q}_{\alpha \beta}^{\prime}\right)$ has genus $n$, $n+1$ being the number of components of $\Gamma_{\{\alpha, \beta\}}$. Further, each curve $x_{i}$ (resp. $y_{i}$ ) bounds a 2-ball $\mathbf{X}_{i}$ of $\mathbb{Q}_{\alpha \beta}$ (resp. $\mathbf{Y}_{i}$ of $\left.\mathbb{Q}_{\alpha \beta}^{\prime}\right)$, and $\mathbb{Q}_{\alpha \beta}$ (resp. $\mathbb{Q}_{\alpha \beta}^{\prime}$ ), cut open along all the 2-balls $\mathbf{X}_{i}$ (resp. $\mathbf{Y}_{i}$ ), splits into the disjoint union of two 3-balls. It follows that $\mathbb{Q}_{\alpha \beta}$ (resp. $\mathbb{Q}_{\alpha \beta}^{\prime}$ ), cut open along all the $\mathbf{X}_{i}$ (resp. $\mathbf{Y}_{i}$ ), but one, arbitrarily chosen, is a 3-ball. The proof is therefore complete.

Lemma 4. Let $\left(\mathbb{Q}, \mathbb{Q}^{\prime}\right)$ be a Heegaard splitting of $M_{3}, F=\partial \mathscr{Q}=\partial \mathbb{Q}^{\prime}$ its Heegaard surface. Then there exists a crystallisation $(\Gamma, \gamma)$ of $M_{3}$ which regularly imbeds in $F$.

Proof. Let $\left(F, \mathbf{x}=\left(x_{1}, \ldots, x_{n}\right), \mathbf{y}=\left(y_{1}, \ldots, y_{n}\right)\right)$ be a Heegaard diagram, arising from $\left(\mathbb{Q}, \mathbb{Q}^{\prime}\right), n$ being the genus of the splitting. It becomes useful, in the construction of $(\Gamma, \gamma)$, to take the usual representation of the diagram in the ordinary euclidean space (see [S]).

For, let $S^{2}$ be the 2-sphere, represented as the $(x, y)$-plane, plus a point at infinity. For every integer $r$, let $P_{r}=(r,-1), P_{r}^{\prime}=(r, 1), X_{r}, \mathfrak{X}_{r}^{\prime}$ the balls of radius $1 / 4$ and centers at $P_{r}$ and $P_{r}^{\prime}$ respectively, $\mathscr{X}_{r}, \mathscr{X}_{r}^{\prime}$ their interiors, $\mathfrak{x}_{r}, \mathfrak{x}_{r}^{\prime}$ their boundaries. By $\Lambda_{2 n}$ is meant the surface $S^{2}-\cup_{r=1}^{n} \mathscr{X}_{r}-\cup_{r=1}^{n} \mathscr{X}_{r}^{\prime}$. Now let $f: \Lambda_{2 n} \rightarrow F$ be a map, one-to-one everywhere, except that each point of $x_{r}$, $r \in \Delta_{n}-\{0\}$, has two points, one of $\mathfrak{x}_{r}$ and one of $\mathfrak{x}_{r}^{\prime}$, as inverse image. 
Also let $\Lambda_{+}$(resp. $\Lambda_{-}$) be the subset of $\Lambda_{2 n}$ consisting of all the points with nonnegative (resp. nonpositive) ordinate, plus the point at infinity.

By isotoping, if necessary, the y-curves, we can suppose that the following three conditions hold.

(a) For every $i \in \Delta_{n}-\{0\}, f^{-1}\left(y_{i}\right)$ is the disjoint union of a finite set of arcs $\left\{a_{i}^{j}\right\}$, each meeting a circle only at its endpoints.

(b) Let $a_{i}^{j}$ be anyone of the previous arcs, and $\mathfrak{x}_{0}$ the $x$-axis plus the point at infinity; then $a_{i}^{j} \cap \Lambda_{+}$(resp. $a_{i}^{j} \cap \Lambda_{-}$), if nonempty, is the disjoint union of a finite set of arcs $\left\{\mathfrak{b}_{i h}^{j}\right\}$ (resp. $\left.\left\{c_{i k}^{j}\right\}\right)$, none of which meets $\mathfrak{x}_{0}$ at an inner point.

Each circle $\mathfrak{x}_{r}$ (resp. $\mathfrak{x}_{r}^{\prime}$ ), $r \in \Delta_{n}-\{0\}$, is split by the endpoints of the arcs $a_{i}^{j}$ into the union of a finite set of arcs $\left\{\delta_{r s}\right\}$ (resp. $\left\{\delta_{r s}^{\prime}\right\}$ ). Let $\mathcal{E}^{\prime}$ be the union of the families of arcs $\left\{a_{i}^{j}\right\},\left\{\delta_{r s}\right\},\left\{\delta_{r s}^{\prime}\right\}$, and $\mathcal{V}^{\prime}$ the set of their endpoints.

By condition (a), we can consider the pseudograph $\Gamma^{\prime}=\left(V^{\prime}, E^{\prime}\right)$ (multiple edges and loops may occur), where the vertex-set $V^{\prime}$ is in bijection with $\mathcal{V}^{\prime}$, and two vertices $v, w$ are joined by an edge of $E^{\prime}$ iff the corresponding elements of $\mathcal{V}^{\prime}$ are the endpoints of the same arc of $\varepsilon^{\prime}$.

With a slight abuse of notation, we can suppose $\Gamma^{\prime}$ imbedded in $\Lambda_{2 n}$, by identifying $\left(V^{\prime}, E^{\prime}\right)$ with $\left(\mathcal{V}^{\prime}, \mathcal{E}^{\prime}\right)$. With the prior convention, we can state the third condition:

(c) None of the connected components of $\Gamma^{\prime}$ lies entirely in $\Lambda_{+}$or $\Lambda_{-}$.

Let $\left\{\delta_{0 m}\right\}$ be the arcs into which the circle $x_{0}$ is subdivided by the endpoints of the arcs $\left\{\mathfrak{b}_{i h}^{j}\right\}$ and $\left\{\mathfrak{c}_{i k}^{j}\right\}$. By conditions $(\mathrm{b}),(\mathrm{c})$, we can consider a new pseudograph $\Gamma^{\prime \prime}=\left(\mathcal{V}^{\prime \prime}, \mathcal{E}^{\prime \prime}\right)$, where $\mathcal{E}^{\prime \prime}$ is the union of the families of arcs $\left\{\mathfrak{b}_{i h}^{j}\right\},\left\{c_{i k}^{j}\right\},\left\{b_{r s}\right\}$, $\left\{\mho_{r s}^{\prime}\right\}$, and $\mathcal{V}^{\prime \prime}$ the set of their endpoints.

Set $\overline{\mathcal{V}}=f\left(\mathcal{V}^{\prime \prime}\right), \overline{\mathcal{E}}=f\left(\mathcal{E}^{\prime \prime}\right)$. Again a pseudograph $\bar{\Gamma}=(\bar{V}, \bar{E})$, with $(\bar{V}, \bar{E})$ identified with $(\overline{\mathcal{V}}, \overline{\mathcal{E}})$, can be considered.

Observe that the result of the previous construction is the adding of a new cycle $x_{0}=f\left(x_{0}\right)$ to the family $\mathbf{x}$, and the rearrangement of the $y$-curves, in order to get $\bar{\Gamma}$, imbedded in $F$, and whose inverse image under $f$ satisfies properties (a)-(c).

Now let $Q_{r}=(-1, r), Q_{r}^{\prime}=(1, r), \mathscr{Y}_{r}, \mathscr{Y}_{r}^{\prime}, \mathscr{\mathcal { Y }}_{r}, \mathscr{\mathcal { Y }}_{r}^{\prime}, \mathfrak{y}_{r}, \mathfrak{y}_{r}^{\prime}, \Lambda_{2 n}^{\prime}, f^{\prime}: \Lambda_{2 n}^{\prime} \rightarrow F, \Lambda_{+}^{\prime}$, $\Lambda_{-}^{\prime}$ defined as below, simply by exhanging the $x$-axis with the $y$-axis. A perfectly analogous construction can be done, starting from $\bar{\Gamma}$, changing the rôle of the sets $x$ and $y$. The result is a multigraph $\Gamma^{\prime \prime \prime}=\left(\mathcal{V}^{\prime \prime \prime}, \mathcal{E}^{\prime \prime \prime}\right)$, imbedded in $\Lambda_{2 n}^{\prime}$, and a multigraph $\Gamma=(V, E)$, regular of degree four, with $(V, E)$ identified with $\left(f^{\prime}\left(\mathcal{V}^{\prime \prime \prime}\right)\right.$, $\left.f^{\prime}\left(\mathcal{E}^{\prime \prime \prime}\right)\right)$.

Observe that $\Gamma$ can be obtained from $\bar{\Gamma}$ simply by adding a suitable cycle $y_{0}$ to the family of cycles, whose images onto $F$ are the y-curves.

In order to define the colouring $\gamma: E \rightarrow \Delta_{3}$, it suffices to consider the two representations of $\Gamma$ on $\Lambda_{2 n}^{\prime}$ and $\Lambda_{2 n}$, by means of the maps $f^{\prime}, f$ respectively. The first is $\Gamma^{\prime \prime \prime}=\left(V^{\prime \prime \prime}, \mathcal{E}^{\prime \prime \prime}\right)$ while the second, $\Gamma^{\prime \prime \prime \prime}$ say, is obtained from $\Gamma^{\prime \prime}$ by adding the inverse image under $f$ of the cycle $y_{0}$.

Now, label by " 0 " (resp. by "2") all the edges of $\Gamma^{\prime \prime}$, lying in $\Lambda_{+}^{\prime}$ (resp. $\left.\Lambda_{-}^{\prime}\right)$ but not belonging to the cycles $\mathfrak{y}_{0}, \mathfrak{y}_{r}, \mathfrak{y}_{r}^{\prime}, r \in \Delta_{n}-\{0\}$. Analogously, label by " 1 " 
(resp. by "3") all the edges of $\Gamma^{\prime \prime \prime \prime}$, lying in $\Lambda_{+}$(resp. $\Lambda_{-}$), but not belonging to the cycles $\mathfrak{x}_{0}, \mathfrak{x}_{i}, \mathfrak{x}_{i}^{\prime}, i \in \Delta_{n}-\{0\}$.

The pair $(\Gamma, \gamma)$, where $\gamma$ is the induced colouring on the edges of $\Gamma$, is a 4-coloured graph, whose realisation on $F$ provides a regular inbedding i: $|\Gamma| \rightarrow F^{4}$ Moreover, condition (c) proves that $(\Gamma, \gamma)$ is a contracted graph.

By the construction itself, $(\Gamma, \gamma)$ turns out to be a 3-manifold crystallisation, as each of the four subgraphs $\Gamma_{\hat{c}}, c \in \Delta_{3}$, can be regularly imbedded in $\Lambda_{+}, \Lambda_{-}, \Lambda_{+}^{\prime}$, $\Lambda_{-}^{\prime}$, and therefore $\rho\left(\Gamma_{\hat{c}}\right)=0$, for every $c \in \Delta_{3}$ (compare [ $\mathbf{P}_{3}$, Proposition 10] or $\left[\mathbf{G}_{4}\right.$, Corollary $\left.\left.16-\mathrm{e}^{\prime}\right]\right)$.

We have only to show that $(\Gamma, \gamma)$ is a crystallisation of $M_{3}$. For, let $\left\{\xi_{0}, \ldots, \xi_{n}\right\}$ (resp. $\left\{\xi_{0}^{\prime}, \ldots, \xi_{n}^{\prime}\right\}$ ) be the set of connected components of the subgraph $\Gamma_{\{0,2\}}$ (resp. $\Gamma_{\{1,3\}}$ ), whose image on $F$ is $\tilde{\mathbf{x}}=\mathbf{x} \cup\left\{x_{0}\right\}$ (resp. $\tilde{\mathbf{y}}=\mathbf{y} \cup\left\{y_{0}\right\}$ ).

Thus, $(F, \tilde{\mathbf{x}}(\hat{0}), \tilde{\mathbf{y}}(\hat{0}))$ is (equivalent to) the original Heegaard diagram $(F, \mathbf{x}, \mathbf{y})$ of $M_{3}$. The proof of Lemma 4 is thereby complete.

Figures 1(a), 1(b) and 1(c) illustrate the construction of Lemma 4 applied to the classical Heegaard diagram of the Poincaré space (compare [Po]), which is also reproduced in [R, p. 245].

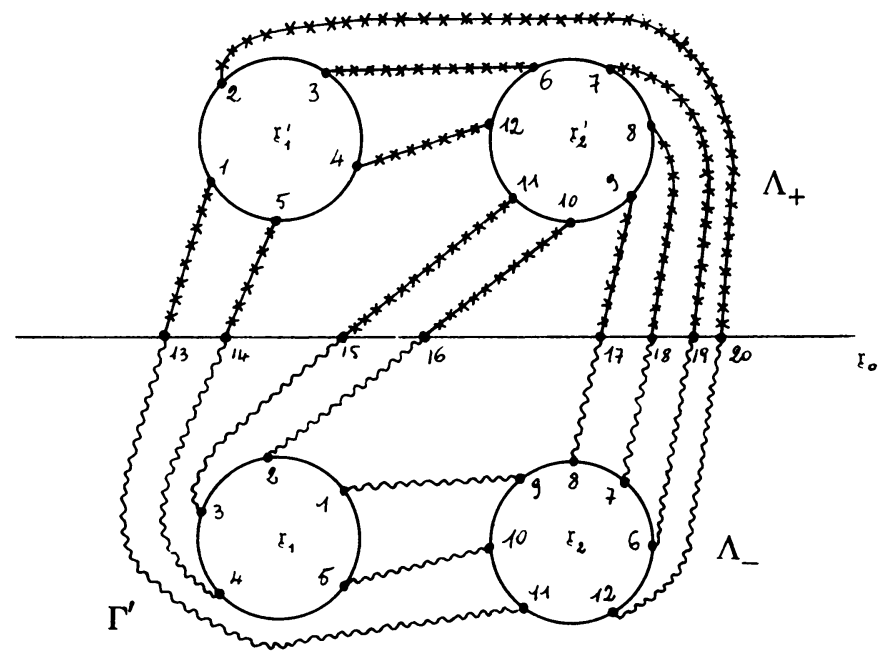

FIGURE 1(a)

Proof of Theorem B. This is an easy consequence of the previous two lemmas.

REMARK. Lemmas 3 and 4 give an algorithm to get a crystallisation from a Heegaard diagram of a closed 3-manifold, and conversely.

\footnotetext{
${ }^{4}$ In the terminology of $\left[G_{4}\right], i$ is induced by the fundamental cyclic permutation of $\Delta_{3}$.
} 


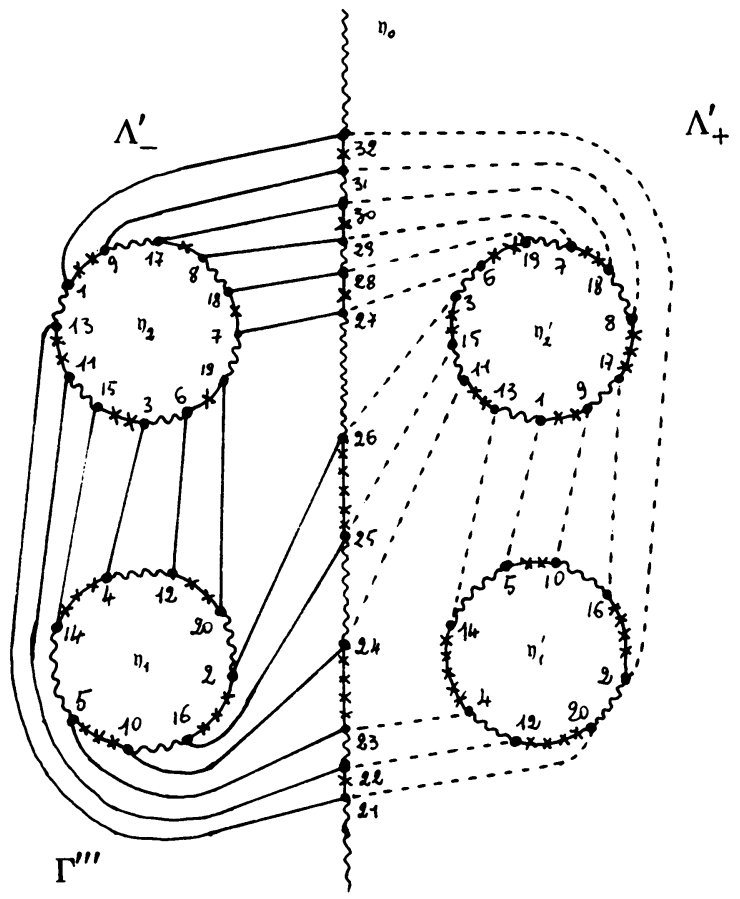

Figure 1(b)

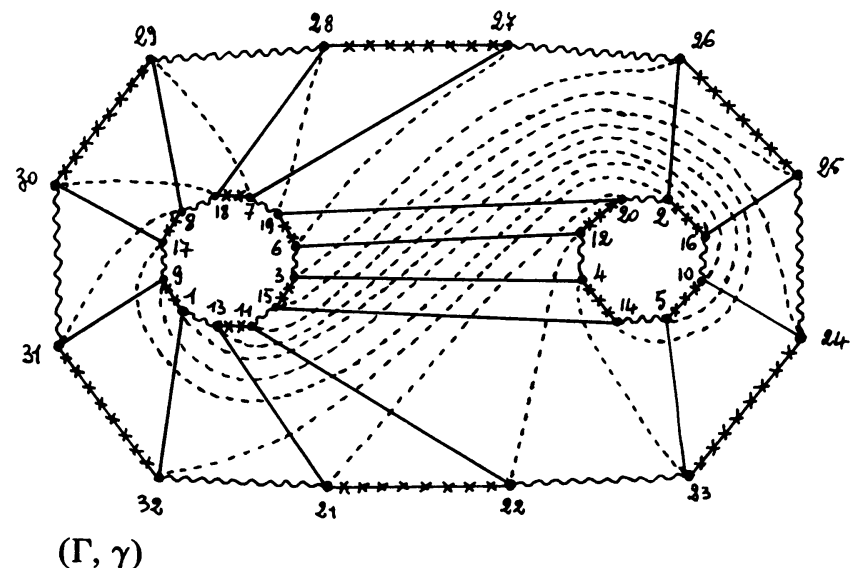

FiguRE 1(c)

4. A geometric approach. Let $M_{n}$ be a closed PL $n$-manifold, $K$ a contracted triangulation of $M_{n}, v_{0}, \ldots, v_{n}$ the $n+1$ vertices of $K, \mathrm{Sd} K$ its first barycentric subdivision. If $A^{n}$ is an $n$-simplex of $K, i, j$ two different elements of $\Delta_{n}$, let us call $A^{n-2}(\hat{i}, \hat{j})$ the $(n-2)$-face of $A^{n}$ not containing the two vertices $v_{i}$ and $v_{j}$.

Now, for every cyclic permutation $\varepsilon=\left(\varepsilon_{0}, \ldots, \varepsilon_{n}\right)$ of $\Delta_{n}$, define $H_{\varepsilon}$ to be the subcomplex of $\mathrm{Sd} K$, obtained as follows: 
(a) for every $n$-simplex $A^{n}$, and for every $i \in \mathbb{Z}_{n+1},{ }^{5}$ consider the barycenter $\underline{a}\left(\hat{\varepsilon}_{i}, \hat{\varepsilon}_{i+1}\right)$ of $A^{n-2}\left(\hat{\varepsilon}_{i}, \hat{\varepsilon}_{i+1}\right)$; then call $\underline{a}\left(\hat{\varepsilon}_{i}\right)$ the barycenter of the $(n-1)$-face opposite to the vertex $v_{\varepsilon}$;

(b) consider the 1-sphere $\Sigma$, consisting of all the 1-simplexes of $\operatorname{Sd} A^{n}$, joining $\underline{a}\left(\hat{\varepsilon}_{i}\right)$ with either $\underline{a}\left(\hat{\varepsilon}_{i}, \hat{\varepsilon}_{i+1}\right)$ or $\underline{a}\left(\hat{\varepsilon}_{i-1}, \hat{\varepsilon}_{i}\right), i$ being an integer modulo $n+1$;

(c) set $\mathscr{D}=\underline{a} * \Sigma, \underline{a}$ being the barycenter of $A^{n}$, and define $H_{e}$ to be the union of all the 2-balls $\mathscr{D}$ 's so obtained.

It is easy to see that the space $F_{e}=\left|H_{e}\right|$ is a closed surface, PL-imbedded in $M_{n}$. We shall call each $F_{\varepsilon}$ a regular surface of $M_{n}$ associated to $K$ and $\varepsilon$.

Observe that, for $n=3$, each regular surface is actually a Heegaard surface, and conversely.

Now set $\tilde{\mathcal{G}}\left(M_{n}\right)=\min \left\{g(F) \mid F\right.$ is a regular surface of $\left.M_{n}\right\}$ where $g(F)$ denotes, as before, the genus of the surface $F$.

Proposition 5. With the previous notations, we have $\tilde{\mathcal{G}}\left(M_{n}\right)=\mathcal{G}\left(M_{n}\right)$.

Proof. Suppose $F=F_{e}(K)$, a regular surface of $M_{n}$ associated to the contracted complex $K$ and the cyclic permutation $\varepsilon$. An easy calculation shows that the Euler characteristic of $F$ is

$$
\chi(F)=\sum_{i \in \mathbf{Z}_{n+1}} q^{n-2}\left(\hat{\varepsilon}_{i}, \hat{\varepsilon}_{i+1}\right)+(1-n) q^{n} / 2
$$

where $q^{n}$ is the number of $n$-simplexes of $K$, and $q^{n-2}(\hat{r}, \hat{s})$ denote the number of $(n-2)$-simplexes of $K$ which do not contain vertices $v_{r}$ and $v_{s}$.

The desired result follows immediately from the definition of regular genus of $M_{n}$, and the formula of $\left[\mathbf{G}_{4}\right.$, Propositions 19, 23].

Remark. A direct geometric proof of Proposition 5 can be accomplished by showing that (i) any crystallisation $(\Gamma, \gamma)$ of $M_{n}$ regularly imbeds into all the regular surfaces associated to $K(\Gamma)$, (ii) if $(\Gamma, \gamma)$ regularly imbeds in a surface $F$, then $F$ can be imbedded in $M_{n}$, so that it becomes a regular surface of it.

Such a construction, for dimension 3 , is exposed in [ $\mathbf{G}_{4}$, Proposition 12].

5. Applications to dimension four. Let $M_{n}$ be a closed orientable (resp. nonorientable) $n$-dimensional manifold; for any contracted triangulation $K$ of $M_{n}$, we shall describe a family of "handlebody decompositions" for $M_{n}$. For every pair of vertices $v_{i}, v_{j}$ of $K, i, j \in \Delta_{n}$, consider the contracted subcomplexes $K_{i j}$ and $K_{i j}$, generated by the vertex-sets $\left\{v_{i}, v_{j}\right\}$ and $\left\{v_{k} \mid k \in \Delta_{n}-\{i, j\}\right\}$ respectively.

Also let $H_{i j}$ be the largest subcomplex of $\operatorname{Sd} K$, disjoint from $\operatorname{Sd} K_{i j} \cup \operatorname{Sd} K_{i j} . H_{i j}$ is a closed orientable (resp. nonorientable, if $n>2)(n-1)$-manifold, which splits Sd $K$ into two complementary subcomplexes, $L_{i j}$ and $L_{i j}$ say, having $H_{i j}$ as common boundary; moreover, they are regular neighbourhoods of $\mathrm{Sd} K_{i j}$ and $\mathrm{Sd} K_{i j}$ respectively, and $\left|L_{i j}\right|$ is an $n$-dimensonal handlebody (i.e. an $n$-ball with 1 -handles attached upon).

${ }^{s} Z_{h}$ denotes the cyclic group of order $h$. 
Suppose now $n=2 k+1$ (resp. $n=2 k$ ), and consider any partition $\left\{\alpha_{0}, \alpha_{1}\right\}$, $\left\{\alpha_{2}, \alpha_{3}\right\}, \ldots,\left\{\alpha_{2 k}, \alpha_{2 k+1}\right\}$ (resp. $\left\{\alpha_{0}, \alpha_{1}\right\},\left\{\alpha_{2}, \alpha_{3}\right\}, \ldots,\left\{\alpha_{2 k}\right\}$ ) of $\Delta_{n}$; then call $\mathbb{Q}_{i}$ the space $\left|L_{2 \alpha_{i}, 2 \alpha_{i}+1}\right|$ for $i \in \Delta_{k-1}$, and $\mathbb{Q}_{k}$ the space $\left|L_{2 \alpha_{k} 2 \alpha_{k}+1}\right|$ or $\left|L_{2 \alpha_{k}}\right|$, according with $n$ being an odd or an even number.

The $(k+1)$-tuple $\left(\mathbb{Q}_{0}, \mathbb{Q}_{1}, \ldots, \mathbb{Q}_{k}\right)$ of handlebodies satisfies the following properties.

(a) $\cup_{r \in \Delta_{k}} \mathbb{Q}_{r}=M_{n}$,

(b) for every $j \in \Delta_{k}, \mathscr{Q}_{j} \cap\left(\cup_{i \neq j} \mathbb{Q}_{i}\right)=\partial \mathbb{Q}_{j}$,

(c) if $n=2 k$, then $\mathbb{Q}_{k}$ is an $n$-ball.

It is easy to see that the $(k+1)$-tuple mentioned previously reduces to a Heegaard splitting for dimension three. For this reason, $\left(\mathbb{Q}_{0}, \ldots, \mathbb{Q}_{k}\right)$ will be called a generalized Heegaard decomposition of $\boldsymbol{M}_{\boldsymbol{n}}$. More details are available in $\left[\mathbf{G}_{\mathbf{1}}\right]$.

The following proposition binds the concepts of "regular surface" and of "generalized Heegaard decomposition", in dimension four.

Proposition 6. Suppose $M_{4}$ a closed 4-dimensional manifold. Then, for every regular surface $F$ of $M_{4}$, there exists a generalized Heegaard decomposition $\left(\mathbb{Q}_{0}, Q_{1}, Q_{2}\right)$ of $M_{4}$, such that $F$ is a Heegaard surface of $\mathbb{Q}_{0}$.

Corollary 7. Let $M_{4}$ be a closed orientable (resp. nonorientable) 4-manifold, and let $\mathcal{G}\left(M_{4}\right)=h$, with $h \geqslant 0$ (resp. $\mathcal{G}\left(M_{4}\right)=2 h$, with $h>0$ ). Then $\pi_{1}\left(M_{4}\right)$ is a quotient of the free group on $h$ generators. In particular,

(a) if $\mathcal{G}\left(M_{4}\right)=0$, then $M_{4}$ is simply connected,

(b) if $\mathcal{G}\left(M_{4}\right)=1$ and $M_{4}$ is orientable (resp. $\mathcal{G}\left(M_{4}\right)=2$ and $M_{4}$ is nonorientable), then $\pi_{1}\left(M_{4}\right)$ is a cyclic group.

Proof. Let $(\Gamma, \gamma)$ be a crystallisation of $M_{4}, \varepsilon$ a cyclic permutation of $\Delta_{4}$, and $F$ the regular surface of $M_{4}$, associated with $K(\Gamma)$ and $\varepsilon$. It follows from Proposition 5 that there exists a regular imbedding $\mathbf{i}:|\Gamma| \rightarrow F$. As shown in $\left[\mathbf{G}_{4}\right]$, $\mathbf{i}$ is induced by a cyclic permutation of $\Delta_{4}$, which is exactly $\varepsilon$, as it is not hard to check. We can suppose, w.l.o.g., $\varepsilon$ induced by the fundamental permutation $(0,1,2,3,4)$.

Now, let us consider the generalized Heegaard decomposition $\left(\mathbb{Q}_{0}, \mathbb{Q}_{1}, \mathbb{Q}_{2}\right)$ of $\boldsymbol{M}_{4}$, relative to the partition $\{0,2\},\{1,3\},\{4\}$ of $\Delta_{4}$; also call $\mathbb{Q}$ the 3-dimensional ball-complex, whose 3-balls are the intersections of $\partial Q_{0}$ with each 4-simplex of $K$, and $\mathbb{Q}^{\prime}$ the 2-dimensional ball-complex, whose 2-balls are the (penthagonal) regions of the dual graph of the imbedding $\mathbf{i}$ in $F .|\mathbb{Q}|$ and $\left|\mathbb{Q}^{\prime}\right|$ are obviously homeomorphic to $\partial Q_{0}$ and $F$ respectively.

Now, each 2-ball of $\mathbb{Q}^{\prime}$ is imbedded in a 3-ball of $\mathbb{Q}$, as indicated in Figure 7 of $\left[\mathbf{G}_{4}\right]$; the induced imbedding, $F \hookrightarrow \partial \mathbb{Q}_{0}$, makes $F$ a Heegaard surface of $\partial \mathbb{Q}_{0}$. This completes the proof of Proposition 6.

To prove Corollary 7, we first remark that $\mathbb{Q}_{0}$ is a 4-dimensional handlebody of genus $\lambda$ (i.e. a 4-ball with $\lambda$ 1-handles attached upon), where $\lambda+1$ is the number of 1-simplexes of $K_{02}$, or equivalently, the number of connected components of the subgraph $\Gamma_{\{1,3,4\}}$. Its boundary $\partial \mathscr{Q}_{0}$ is thereby PL-homeomorphic either to 
$\lambda \# S^{1} \times S^{2}$, if orientable, or to $\lambda \# S^{1} \times S^{2}$, if nonorientable. ${ }^{6}$ By the prior proposition, if $g(F)=\mathcal{G}\left(M_{4}\right)$, then $\lambda<h$ in both the orientable and the nonorientable cases. Now, the main result of $\left[G_{3}\right]$ states that $\pi_{1}\left(M_{4}\right)$ admits a presentation with $\lambda$ generators, and the proof is therefore complete.

As an easy consequence of the previous construction, we can state the following.

COROllaRy 8. If $M_{4}$ is a closed nonorientable 4-manifold, then $\mathcal{G}\left(M_{4}\right)$ is an even positive number.

We conjecture that Proposition 6 and Corollaries 7 and 8 can be generalized to all dimensions.

\section{REFERENCES}

[F] M. Ferri, Crystallisations of 2-fold branched coverings of $S^{3}$, Proc. Amer. Math. Soc. 73 (1979), 271-276.

[FG] M. Ferri and C. Gagliardi, Crystallisation moves, Pacific J. Math. (to appear).

[G] C. Gagliardi, Spezzamenti alla Heegaard per varietà n-dimensionali, Boll. Un. Mat. Ital. 13-A (1976), 302-311.

[G $\left.\mathbf{G}_{2}\right] \ldots$ A combinatorial characterization of 3-manifold crystallization, Boll. Un. Mat. Ital. 16-A (1979), 441-449.

[G] How to deduce the fundamental group of a closed n-manifold from a contracted triangulation, J. Combin. Inform. System Sci. 4 (1979), 237-252.

[G] _ Regular imbeddings of edge-coloured graphs, Geom. Dedicata (to appear).

[G] L. C. Glaser, Geometrical combinatorial topology, Vol. I , Van Nostrand Reinhold, New York, 1970.

[Ha] F. Harary, Graph theory, Addison-Wesley, Reading, Mass., 1969.

[He] J. Hempel, 3-manifolds, Princeton Univ. Press, Princeton, N. J., 1976.

[HW] P. J. Hilton and S. Wylie, An introduction to algebraic topology-homology theory, Cambridge Univ. Press, New York, 1960.

[M] J. M. Montesinos, Sobre la representacion de varietades tridimensionales, Mimeographed notes, 1976.

[ $\left.\mathbf{P}_{\mathbf{1}}\right]$ M. Pezzana, Sulla struttura topologica delle varietà compatte, Atti Sem. Mat. Fis. Univ. Modena 23 (1975), 269-277.

$\left[\mathbf{P}_{2}\right]$, Diagrammi di Heegaard e triangolazione contratta, Boll. Un. Mat. Ital. 12-A Suppl. Fasc. 3 (1975), 98-105.

[Po] H. Poincaré, Cinquième complément à l'analysis situs, Rend. Circ. Mat. Palermo 18 (1904), 45-110.

[R] D. Rolfsen, Knots and links, Publish or Perish, Boston, Mass., 1976.

[S] J. Singer, Three-dimensional manifolds and their Heegaard diagrams, Trans. Amer. Math. Soc. 35 (1933), 88-111.

[ST] H. Seifert and W. Threlfall, Lehrbuch der Topologie, Teubner, Leipzig, 1934.

[W] A. T. White, Graphs, groups, and surfaces, North-Holland, Amsterdam, 1973.

Istituto Matematico “G. Vitali”, Universiti di Modena, Via Campi 213/B, 41100 Modena, ITALY

${ }^{6} \lambda \# S^{1} \times S^{2}$ (resp. $\lambda \# S^{1} \times S^{2}$ ) denotes the connected sum of $\lambda$ copies of $S^{1} \times S^{2}$ (resp. of the nonorientable 2-sphere bundle over $S^{1}$ ). 